tris:

TRANS---No 8-· 2004 SECCIÓN - 37-59
En este artículo se analiza la variedad de cambio de código inglésespañol utilizada por el pueblo chicano. Tras realizar un acercamiento a este concepto, se encuadra como elemento importante dentro de la literatura chicana. A continuación, se estudian las estrategias de cambio de código usadas por la autora Sandra Cisneros en su última novela, Caramelo or Puro Cuento, y se comparan con las utilizadas en la versión española de la obra, realizada por Liliana Valenzuela. En el primer caso, destaca la presencia de términos en español seguidos de su correspondiente traducción al inglés o de expresiones sin traducción, cuyo significado se deduce a través del contexto. El español también se encuentra en interjecciones o vocativos. En la traducción se compensa este rasgo lingüistico adoptando estrategias similares a las del original, y añadiendo términos ingleses a la narración en español.

PALABRAS CLAVE: cambio de código, spanglish, literatura chicana, traducción literaria.

\title{
Estrategias de cambio de código y su traducción en la novela de Sandra Cisneros Caramelo or Puro
}

Nieves Jiménez CarRa

Universidad de Malaga
This paper deals with the variety of English-Spanish code-switching spoken by the Chicano people. I shall approach this concept, together with its importance within the Chicano literature, and analyse the strategies of code-switching used by author Sandra Cisneros in her last novel, Caramelo or Puro Cuento. Also, I will compare them with those present in the Spanish translation of the work, carried out by Liliana Valenzuela. The original text is mainly characterized by the occurrence of Spanish terms followed by their English translations. Some other expressions, however, go untranslated, and their meanings can be inferred from the context. Spanish interjections and vocatives also appear. In the translated version, as a compensation, similar codeswitching strategies are employed and English terms are added to the Spanish narration.

KEYWoRDS: code-switching, Spanglish, Chicano literature, literary translation. 


\section{INTRODUCCIÓN AL CAMBIO DE CÓDIGO} INGLÉS-ESPAÑOL: LOS CHICANOS EN EE.UU.

El cambio de código o code-switching es la intercalación dentro de un mismo discurso de elementos léxicos y/o sintácticos pertenecientes a dos idiomas. En el caso que nos ocupa, las lenguas en contacto que se entremezclan y se combinan hasta crear un nuevo sistema de comunicación, ${ }^{2}$ son el inglés y el español, y, en concreto, la variedad de cambio de código utilizada por los chicanos, es decir, los mexicanos o las personas de origen mexicano que viven dentro de las fronteras de Estados Unidos?. Este fenómeno, sin embargo, no es exclusivo de este colectivo, y se extiende a otros grupos hispanos (cubanos, puertorriqueños, etc.), que habitan dentro del país norteamericano.

El cambio de código nace, fundamentalmente, de la necesidad y voluntad de los hablantes de mantener vivas sus tradiciones (en este caso, representadas por el idioma español, aunque también harán lo propio con el resto de elementos culturales), y de integrarse en

I Al igual que la mayoría de los autores, opto aquí por usar la designación «cambio de código" en su acepción más amplia. Sin embargo, es necesario aclarar que existe un nombre específico para el tipo de fenómeno que protagoniza este estudio: «mezcla de código». En un sentido estricto, el primer caso sólo englobaría aquellos discursos en los que se pasa directamente de un idioma a otro, mientras que el segundo se referiría a los que, aun desarrollándose en una lengua, incluyen términos, interjecciones o estructuras propias de otra.

2 Sigo a Raquel León Jiménez (2003; 18), en cuanto a la denominación del discurso resultante de un cambio de código, cuando afirma que «el uso del término lengua no debe entenderse en sentido estricto y debería sustituirse por el de comportamiento lingüistico o conducta verbalm. Por tanto, siempre que se mencione en este trabajo la lengua chicana, se entenderá conforme a esta aclaración.

3 La Real Academia Española recomienda escribir $\operatorname{con} x$ el topónimo México y todos sus derivados, por ser la grafía utilizada en ese país y en el resto de Hispanoamérica. Se trata de un arcaísmo ortográfico del español, que se conservó en la variante de América, y que derivó en España en la grafía $j$, que, aun así, sigue siendo correcta (www.rae.es). Por tanto, será ésa la forma que se utilice en este trabajo. la comunidad en la que ahora viven ${ }^{4}$. Según Baker (1975: 171), tanto los individuos que pertenecen al grupo étnico (en nuestro caso, el chicano), como los que lo observan desde fuera, identifican el idioma como un símbolo de la comunidad y de su entorno cultural. Además, el grado de conocimiento de la nueva lengua o de la nueva cultura simboliza el estatus que el individuo tiene en la sociedad a la que ahora pertenece.

En el caso de la comunidad chicana, el uso del español y, por consiguiente, el apego a la cultura y las tradiciones mexicanas, se ve reflejado en el hecho de que son pocos los que abandonan el idioma y sólo hablan inglés, incluso en un contexto familiar. Esto, como declara Aurelio M. Espinosa (1975: 101), "speaks eloquently for the tenacity and vigor of Spanish tradition and culture». De hecho, la lengua constituye un elemento fundamental de la cultura chicana, tanto si se trata del español como de la lengua chicana (Eguíluz Ortiz de Latierro, 2000: 102)5. A pesar de que el inglés «dominates Spanish in the text [...], Spanish still resists, in the same way that Mexican culture remains in the United States, without being completely erased" (Martín Junquera, 2000: 228). A este respecto, algunos autores chicanos confiesan tener más facilidad para usar el inglés en sus obras. Sandra Cisneros admite que ume hacen falta las palabras en español [para escribir una novela en este idioma]. Tengo carencias de determinados aspectos del español, porque lo aprendí de niña» (Lucio, 2003). Rudolfo Anaya advierte, sin embargo, que «siempre que puede acude al español para matizar los significados concretos y únicos de sus pasajes que, de otra forma, resultarían descargados de la

4 En concreto, el cambio de código «can be considered a socio-geographical variation posing problems because it is used [...] in every Chicano text, to impose some kind of individual cultural identity and to convey important features [...]” (Martín Junquera, 2000: 231).

5 Este autor llama a la lengua chicana einglés característico chicanon. 
profundidad hispana que llevan dentro de sí sus personajes» (Eguíluz Ortiz de Latierro, 2000: 103). También, "Alurista y Ricardo Sánchez son pioneros en la utilización del "caló", basado en el paso del inglés al español dentro de la misma frase, párrafo y obra, [...] la justificación es sencilla: ésta es la lengua que utilizan la mayoría de los chicanos en su vida diaria" (Eguíluz Ortiz de Latierro, 2000: 103) ${ }^{6}$.

Sin embargo, y a pesar de la fuerza con la que el español sigue presente en la vida de los chicanos, las nuevas generaciones poseen un noción bastante pobre del idioma. Este hecho se explica si tenemos en cuenta que el niño chicano estudia por lo general en un ambiente angloparlante, y es en este entorno académico donde aprende a desenvolverse y donde adquiere un tipo de vocabulario y un estilo lingüístico más elevado que el del español, que sólo habla en un ambiente coloquial y familiar.

En este caso, en el que el inglés y el español están en contacto permanente y su uso se determina por el contexto en el que el hablante se encuentra, podemos hablar de diglosia, un término acuñado por Ferguson "to refer to situations where either two varieties of the same language or two different languages are coextensively used in the same society, i.e. one colloquial variety (or varieties) for everyday affairs, and one more respected variety used for writing and formal affairs» (Peñalosa, 1975: 164). Así, François Grosjean afirma que

[...] in their everyday lives, bilinguals find themselves at various points along a situational continuum which induce a particular language mode. At one end of the continuum, bilinguals are in a totally monolingual language mode: they are speaking (or writing) to monolingual speakers of either language $\mathrm{A}$ or

6 Raquel León Jiménez (2003: 18) afirma que «junto a la denominación "Spanglish" pueden encontrarse otros vocablos, algunos de índole peyorativa, [...] como "pocho", "caló" o "Tex-Mex" [...]. [L]a mayoría de los chicanos prefieren nombrar a su sistema de comunicación como la "lengua chicana"». of language $B$ and therefore have to restrict themselves to just one language (A or B). At the other end of the continuum, they are with bilinguals who share their two languages $(A$ and $B$ ) and with whom they normally mix languages (code-switching and borrow), they are here in a bilingual language mode. [...] (Grosjean, 1995: 261-262) (el énfasis es mío).

Sin embargo, también en contextos no coloquiales podemos encontrar ejemplos de cambio de código, como los abanderados por la revista norteamericana Latina: Magazine for Hispanic Women: «[Penélope Cruz] is one mujer on the move»; "a four-week celebration highlighting the accomplishments and contributions de nuestra comunidad»; «Gente who are practically Latino" "Tego Calderón: the voice of la calle" o «Am I his novia or his mother?» (el énfasis es de la revista) 7 .

Relacionado con el cambio de código se encuentra el fenómeno lingüístico por excelencia en cuanto a la interacción español-inglés: el spanglish. Diez Vegas (1994) lo define como «un "idioma" híbrido formado a partir del español al introducir términos anglosajones sin traducir o traducidos incorrectamente» ${ }^{8}$. En la

7 Todos los ejemplos fueron tomados del número de octubre de 2004 de Latina, consultado en www.latina.org el 25 de octubre de 2004. En el mismo número, una encuesta preguntaba a los cibernautas: «Can you fully embrace your Latina roots without speaking Spanish?m. Las respuestas posibles eran: "Definitely not!», "Absolutely!", «Somewhat" $y$ «Speaking Spanish has nothing to do with it». El 31 de octubre de 2004 , las votaciones eran, respectivamente, las siguientes: $137,64,93$ y 117 votos. Por tanto, el respaldo a la lengua española como raíz importante para mantener la esencia de la identidad como latino llegaba casi al 34\%, aunque también era elevado el porcentaje de personas que no la relacionaba con este hecho.

No me detendré aquí a realizar un análisis exhaustivo de los factores que producen el spanglish, sólo proporcionaré una visión general del fenómeno, con objeto de encuadrarlo dentro del tema de este trabajo. En la web existe abundante información en torno a este tema, sobre todo en la página www.elcastellano.org. También se puede acudir al libro Spanglish: The Making of a New American Language (HarperCollins, 2003), de Ilan Stavans, uno de los máximos defensores del fenómeno, $y$ autor también de un diccionario de spanglish. 
mayor parte de las ocasiones en las que se habla de spanglish, se piensa en un discurso con base española, salpicado de elementos anglosajones, 40 e incluso de "adaptaciones» al español, a veces sin sentido, de términos ingleses (recuérdese el ejemplo por antonomasia: "vacunar la carpeta», traducción literal de to vacuum the carpet). De hecho, la mayoría de autores sólo mencionan esta variante. Así, encontramos afirmaciones como «el spanglish es una invasión del español por el inglés» (González Echeverría, 1997), «unless you speak Spanish, you might not be aware of the controversy over Spanglish" (Paternostro, 2003) o «idioma español hablado con "abundancia de anglicismos" (Seco, Andrés y Ramos, 1999: 4.179). Efectivamente, el término en sí mismo intensifica y da prioridad a la lengua española sobre la inglesa. Sin embargo, no será del spanglish en sentido estricto de lo que se trate en este trabajo, ya que considero que el cambio de código constituye un paso intermedio entre el discurso monolingüe y el spanglish o, si se quiere, se trata de una variante diferente del spanglish tradidional.

De lo anteriormente expuesto, deduzco que el cambio de código, aunque incluido a veces en las definiciones más amplias de spanglish (en el caso de la revista Latina, se llama spanglishism tanto a la frase «My job has me re-frita» como a "Be careful- this street is a Juan Guey! [one-way]»), no puede incluirse de forma plena bajo esta denominación, si tenemos en cuenta la mayoría de sus acepciones. Es cierto que se trata de una mezcla de ambos idiomas, pero con una base inglesa, con estructuras gramaticales y sintácticas que parten de las de la lengua inglesa, y donde se añaden términos en español, para marcar, como he mencionado antes, la pertenencia a un grupo distinto del mayoritario en la sociedad («don't worry, papá», "mis abuelos lived in México for 50 years») 9 . En

9 «En muchas ocasiones, el paso de una variedad a otra se produce dentro del turno conversacional de un efecto, ya Grosjean lo advertía en 1995 cuando diferenciaba entre cambio de código, préstamo y una variedad de este último, que se puede identificar, aplicándolo a nuestro caso, como spanglish:

Code-switching is shifting (switching) completely to the other language for a word, a phrase, a sentence, etc. 'Borrowing' is taking a word or short expression and (usually phonologically or morphologically) adapting it to the base-language. It can also refer to taking the meaning component of a word or expression and drafting it onto a word or series of words in the base-language, but in this case it may be that the underlying processes at work are quite different from those involved in code-switching and outright borrowing. (Grosjean, 1995: 263).

Tanto si nos referimos al cambio de código como al spanglish, se plantea aquí la duda de si semejantes variaciones, distorsiones o evoluciones de la lengua (elijase sustantivo en función de nuestra percepción del fenómeno), son fruto del conocimiento exhaustivo de los dos idiomas en contacto o se deben, más bien, a una incompetencia en ambos. Esta última estaría acompañada del recurso a la otra lengua para expresar lo que no se puede formular, por desconocimiento, en la primera. Huelga decir que los detractores del spanglish y, por ende, del cambio de código, afirman sin ningún atisbo de dudas, que es resultado de la ignorancia del hablante. Así, González Echeverría (sin fecha) dice que «la triste realidad es que el spanglish es básicamente la lengua de los hispanos pobres, muchos de los cuales son casi analfabetos en cualquiera de los dos idiomas [, aunque, por otra parte,] los hispanos educados que hacen otro tanto tienen una motivación diferente:

único hablante, mientras que en otras ocasiones el cambio se produce dentro de los límites de una misma oración, prescindiendo de cualquier pausa sintáctica para separar los códigos empleados» (León Jiménez, 2003: 34-35). 
algunos se avergüenzan de su origen e intentan parecerse al resto usando palabras inglesas y traduciendo directamente las expresiones idiomáticas inglesas». Xosé Castro (2001), por su parte, afirma que «hablamos [...] de una estructura comunicativa, mayormente léxica, constituida, aunque no siempre, sobre carencias en las que, casi inmediatamente, intuimos cierta marginalidad social».

Desde la otra perspectiva, se alega que «el spanglish es una muestra de destreza lingüística" (Ana Celia Zentella, citada por Javier Valenzuela, sin fecha), y algunos llegan tan lejos como para declarar, sin pudor, que «if Spanish purists do not embrace modernity [por "modernity" el autor se refiere al spanglish], Latinos have found a way of entering today's globalized world» (llan Stavans, citado por Paternostro, 2003).

En cuanto al cambio de código en particular, y desde el punto de vista teórico, Pieter Muysken (1995: 177) recuerda que

in the last fifteen years, a large number of studies have appeared in which specific cases of intra-sentential code-switching were analysed from a grammatical perspective, involving a variety of language pairs, social settings and speaker types. It was found that code-switching is a quite normal and widespread form of bilingual interaction requiring a great deal of bilingual competence.

Del mismo modo, Köppe y Meisel (1995: 277) añaden que «the term 'code-switching' more specifically, is used in the almost commonly accepted sense to describe a certain skill of the bilingual speaker that requires pragmatic and grammatical competence in both languages".

De cualquier forma, el uso que hacen los chicanos del cambio de código en su discurso diario nos proporciona la mejor perspectiva para juzgarlo. Lo que en un principio constituía un sistema de comunicación básicamente familiar y coloquial, se ha convertido, además, en la base de una nueva forma de producción literaria.

\section{LA LITERATURA CHICANA. SANDRA CISNEROS Y CARAMELO OR PURO CUENTO}

La identificación con un grupo étnico y cultural diferente al de la mayoría se sitúa, pues, como una de las causas principales de la aparición de la llamada «literatura chicana $»^{\mathrm{T}}$. Su esencia, por tanto, es diversa a la de la literatura norteamericana, aun en el caso de que ambas estén escritas en inglés y de que su base geográfica sea la misma. La historia, sociedad, cultura y lengua de los chicanos, influyen en su producción literaria (Eguíluz Ortiz de Latierro, 2000: 99). Si ya he mencionado en el apartado anterior la presencia de la lengua como símbolo cultural, la familia se sitúa como pilar básico de la cultura chicana, en la que las relaciones entre sus miembros están siempre presentes y constituyen un elemento fundamental de la sociedad. De hecho, Eguíluz Ortiz de Latierro (2000: 102) afirma que «es imposible entender al chicano sin entender la importancia de la familia».

Tal es la relevancia de estos elementos en la vida de los chicanos que «los atributos culturales de la lengua, los valores, las creencias son los elementos que han unido a los chicanos, incluso con sus diferencias dentro de esa cultura [la norteamericana]" (Eguíluz Ortiz de Latierro, 2000: 104).

El cambio de código está presente en muchas de sus obras y, principalmente, sirve para determinar si un personaje está o no está integrado en la nueva cultura, y si esa integración supone una ruptura con su herencia mexicana. Men-

\footnotetext{
Io «Los autores chicanos dan a conocer la identidad de su grupo a través del discurso con el que caracterizan a sus personajes, el cual refleja la conducta verbal de la comunidad con el fin de legitimarla a través de sus publicaciones» (León Jiménez, 2003: II).
}

\section{1}


ciona León Jiménez (2003: 60-61) el caso de Soldierboy, de J. y S. Pérez, donde,

al principio de la obra, el narrador nos informa, en una acotación, de que Frank es un hablante fluido en español [...], pero, desde que vuelve de la guerra, el inglés [...] se convierte en el único sistema que da voz a sus palabras [...]. La temporada que ha pasado fuera de su círculo familiar ha convertido a Frank en un hablante lame [Y añade en una nota a pie, citando a Montes Granado:] El término se aplica a quienes, viviendo en la frontera entre dos culturas, aceptan el código de mayor peso social, en detrimento del suyo propio, hasta alejarse totalmente de éste y su cultura.

En un primer estadio, la literatura chicana nace como una forma de reivindicación social

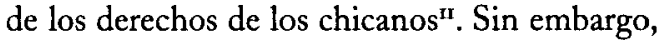
esa imagen de lucha social ha quedado atrás y se ha evolucionado hacia una literatura más abierta y destinada a un público más amplio. Ibarraran Gabilondo (2000: 30-31) confirma que,

en sus primeros momentos, tenía un carácter social de protesta muy fuerte. Hoy en día [...] la literatura ha empezado a perder esa función social que cumplía hasta entonces [...]. Las autoras chicanas [...] están rompiendo las fronteras de su propio grupo, creando una literatura más universal y abierta.

Ciertamente, estas mujeres comparten una cultura y una tradición eminentemente machista que, generalmente, les obliga a quedarse en casa. Por tanto, su obra será una forma

II «[La] efervescencia de una producción artísticoliteraria coincide históricamente con la erupción del movimiento obrero. [...] [E]l movimiento político y la literatura parecen quedar fundidos dentro de una sola dinámica» (Flores, 2000: 145-146). de liberación y de denuncia de su situación ${ }^{12}$. Henriquez Betancor (2000: 173) confirma que las experiencias personales de las chicanas les sirvieron de base para desarrollar su creación literaria, así como para liberarse de la opresión y represión.

La poesía y la novela son los géneros más empleados por las autoras chicanas. La poesía permite a estas mujeres tratar temas sociales. Sin embargo, será en las novelas donde se verán mejor representadas. Las protagonistas de éstas son, casi siempre, mujeres cuyas vidas transcurren en situaciones y entornos muy parecidos a los de las propias escritoras, de modo que, en muchas ocasiones, estas obras tendrán un carácter poco menos que autobiográfico (Ibarraran Gabilondo, 2000: 47-48). Una de las características que suele acompañar a las obras es la presencia de un niño como narrador, en primera persona. En el caso de las dos novelas de Sandra Cisneros, se repite este patrón. En The House on Mango Street (La casa en Mango Street), la protagonista, Esperanza, habla con voz de niña, pero utilizando una ironía propia de un adulto. Por otra parte, en Caramelo or Puro Cuento (Caramelo o puro cuento), se intercalan las narraciones infantiles y adultas del personaje principal, Lala.

Según Martín Rodríguez (2000: 256), la estrategia de usar niños como narradores "permite explotar al máximo la identificación del lector con el protagonista que descubre poco a poco [como le ocurre al receptor] elementos

12 La autora cuya obra se analiza en este artículo, Sandra Cisneros, afirma en dos entrevistas: "Tuve que romper con muchas de las esperanzas de mis padres, que no podian comprender que saliera de mi casa sin un marido que me diese hijos» (Lucio, 2003) y "For a girl in a Mexican family, your life too many times becomes a choice between being the 'Virgen de Guadalupe' or a 'puta', 'a wild thing", she says. "I knew it was not my 'destino', my destiny, to follow my father's wishes and live quietly at home until I found a husband and moved to his home so he could take care of me" (Guinn, 2002). En estas últimas declaraciones se puede, asimismo, observar el cambio de código que Cisneros utiliza en su propio discurso. 
que le eran desconocidos de su ambiente y/o de su culturam. Ésta es la forma que, en estos casos, el autor utiliza para acercar la trama de la obra al público.

\subsection{La autora: Sandra Cisneros}

Nacida en Chicago en 1954, la vida de Sandra Cisneros ha estado ligada a México desde su infancia. Su padre era mexicano y su madre chicana. El hecho de ser la única hija de un total de siete hermanos también influyó, como ya se ha apuntado, en la reacción familiar al camino literario elegido por Cisneros.

Después de graduarse en la Loyola University of Chicago en 1976, y de estudiar en la Universidad de Iowa, comenzó a escribir artículos en revistas literarias (algunas de ellas hispanas, como la Revista Chicano-Riqueña) y de poesía. Pero no fue hasta 1984 cuando concluyó la primera de las dos novelas que ha escrito hasta el momento: The House on Mango Street ( $L a$ casa en Mango Street), publicada inicialmente en Arte Publico Press de Houston, y con la que obtuvo un premio de la fundación Before Columbus en 1985. La poesía ha ocupado, sin embargo, la mayor parte de su carrera literaria. Sus obras poéticas son Bad Boys (1980), My Wicked Wicked Ways (1987) y Loose Woman (1994). También relevante resulta la colección de historias Woman Hollering Creek and Other Stories (El arroyo de la llorona) (1991), que en la particularidad de su título recuerda la convivencia entre el inglés y el español (en este caso, en la localidad tejana de San Antonio, donde Cisneros vive actualmente), que constituye una presencia constante en la vida y obra de la autora ${ }^{{ }^{3}}$. E1 libro infantil Hair/Pelitos (1994) es otra de las producciones de Cisneros hasta que,

I3 McQuien y Hochmeister (2003) explican que «the title [de esta obra], incidentally comes from the awkward English translation of "la llorona" (the moaning woman), the name of a creek located east of San Antonio toward Seguin». en el 2002, publica Caramelo or Puro Cuento (Caramelo o puro cuento).

Sandra Cisneros es una mujer vivaz, habladora y a veces controvertida. Es conocida entre muchos de sus lectores la polémica que originó al pintar su casa en el barrio King William de San Antonio de color violeta. El ayuntamiento consideraba que el color no estaba acorde con el espíritu histórico de la vecindad. Ella argumentó que, según la tradición tejana, este color era histórico. Cito este ejemplo, porque lo considero buen indicativo de la personalidad de la autora, $y$, además, para insistir una vez más en la importancia que la comunidad chicana otorga a sus tradiciones, a sus ancestros y a su idioma. Sirva como muestra de esto el siguiente extracto de su artículo de «autodefensa»:

I thought I had painted my house a historic color. Purple is historic to us. It goes back a thousand years or so to the pyramids. It is present in the Nahua codices, book of the Aztecs, as is turquoise. [...] But we are people sin documentos. We don't have papers. Our books were burned in the conquest, and ever since, we have learned to keep quiet, to keep our history to ourselves, to keep it alive generation to generation by word of mouth, perhaps because we feared it would be taken away from us again. [...] The issue is bigger than my house. The issue is about historical inclusion. I want to paint my house a traditional color. But I don't think it unreasonable to include the traditions of los tejanos who had a great deal to do with creating the city of San Antonio we know today" (Cisneros, 1997).

$\mathrm{El}$ hecho de que la autora recurra al español en pleno discurso inglés («sin documentos», «los tejanos» y, en otro lugar del artículo, «look at my casita"), es, probablemente, una forma de provocación ante un hecho que ella considera discriminatorio hacia su grupo (he aquí, sobre todo, la «defensa» que mencionaba antes).

En cuanto a su forma de hablar y de escribir, 
Jeff Guinn (2002) es claro al comentar que:

Sandra Cisneros talks like she writes, which means that, whether you're reading her new novel "Caramelo" or speaking to her in person, you're not going to slide routinely from Point $A$ to Point $B$ and onward in predictable order. [...] Cisneros gives the impression of being all over the topical map until at some moment or other, it becomes clear she's been deftly binding apparently unrelated things together and making perfect, entertaining sense.

Estas caracteristicas lingüísticas y literarias se constatan en la obra que estudiamos en este trabajo.

\subsection{La obra y su traducción}

Casi veinte años distan entre la publicación de la primera y la segunda (y, hasta el momento, última) novela de Sandra Cisneros. Caramelo or Puro Cuento es una novela escrita para los inmigrantes y dedicada al padre de la autora ${ }^{14}$. Cisneros la comenzó con la intención de contar una historia basada en un viaje a México de su familia y las de sus tíos, y con la idea de que no fuera extensa. Sin embargo, afirma, «when I tried to write about this small foray, I could never get to a key scene» (Guinn, 2002). Para contar la historia de su padre, tenía antes que contar la de sus abuelos, y la de su madre. De

I4 El padre de Cisneros era tapicero, como el padre de la protagonista de la novela. La primera parte del título, Caramelo, recuerda, tanto a un tipo de rebozo (una especie de manto mexicano) de seda y a rayas, como a una niña que la autora vio una vez en Acapulco y que, según ella, tenía la piel del color del caramelo. La segunda parte, Puro Cuento, recuerda la confusión entre lo real y lo inventado en la obra: "The truth, these stories are nothing but story, bits of string, odds and ends found here and there, embroidered together to make something new. I have invented what I do not know and exaggerated what I do to continue the family tradition of telling healthy lies. If, in the course of my inventing, I have inadvertently stumbled on the truth, perdónenme. To write is to ask questions. It doesn't matter if the answers are true, or puro cuenton (Cisneros, 2002). esta forma, cambió el enfoque de la obra, y, utilizando la voz de la protagonista, Lala, y las incursiones en la narración de la abuela (que interrumpe a la nieta para hacer pequeños incisos), unió todas estas historias bajo la misma trama.

Lala se presenta al lector en tres etapas de su vida: como niña, como adolescente y como adulta. Con ella, nos acercamos a su familia, a las anécdotas de su vida y a las relaciones de amor y odio que se establecen entre sus miembros.

A pesar de incluir elementos que podrian identificarse con la vida de Cisneros, no se trata de una novela autobiográfica, sino, más bien, de retazos basados en la vida de la familia de la autora, con elementos en común (como, por ejemplo, un viaje de los tres hermanos a México o la mala relación entre la abuela y la madre de Lala, y las de Sandra Cisneros), que sirven de excusa para recorrer, a la vez, la historia de México y Estados Unidos. De hecho, Cisneros advierte: «This is not a family memoir [...]. A lot of people are going to take it as that. They always take my writing as factual. It's fact-based, but I just use it as a springboard" (Newman, 2002). Curiosamente, no sólo sus admiradores creen su obra como real, sino también su propia familia: "Her mother has read the book only in dribs and drabs, "like arsenic", Cisneros said, perhaps because she's afraid of how the mother in the book is depicted, even though it is not really her» (Newman, 2002).

Una de las características de la obra, que la diferencian de la mayoría de este tipo, es la presencia de copiosas notas a pie de página. Aportan no sólo datos históricos, sino también anecdóticos, así como explicaciones de elementos inequívocamente culturales, como es el caso del lenguaje de las telenovelas, que se adivina en numerosos diálogos de la obra. Como explicación a uno de ellos, encontramos la siguiente nota: 
«-¿Qué intentas ocultar? - ¿Por qué eres tan cruel conmigo? - Te encanta hacerme sufrir. ¿Por qué me mortificas? Say any of the above, or say anything twice, slower and more dramatic the second time 'round, and it will sound like the dialogue of any telenovela" (TO: 15) is.

«¿Qué intentas ocultar? - ¿Por qué eres tan cruel conmigo? -Te encanta hacerme sufrir. - ¿Por qué me mortificas? -Di cualquiera de las frases anteriores, o di cualquier frase dos veces, en forma más lenta y más dramática la segunda vez, y sonará como el diálogo de cualquier telenovela» (TM: 30).

Con este tipo de acotaciones, que ilustran el entorno histórico y cultural de los protagonistas, Cisneros logra crear un ambiente óptimo en el que es difícil perderse en malentendidos culturales, puesto que ella clarifica cualquier situación que al lector le pueda resultar desconocida. Completa esta información una cronología con la que concluye la obra. También es frecuente hallar fragmentos de canciones, por lo general en español y sin traducir, excepto dos de ellas: La Petenera (TO: 50-51) y Júrame (TM: 182-183).

Dada la complejidad que entraña la redacción de la obra, la mezcla de dos idiomas durante la totalidad de la narración y, además, los escollos culturales que planteaba, Sandra Cisneros eligió como traductora de Caramelo or Puro Cuento al español a Liliana Valenzuela, una escritora y antropóloga, natural de México, $y$ con residencia en Texas. Autora y traductora son amigas $y$ trabajaron juntas en la traducción durante mucho tiempo. El texto final, sin embargo, es responsabilidad de Valenzuela. Como advierte Cisneros, y como comprobare-

I5 Las páginas aludidas tras los ejemplos corresponden a las siguientes ediciones: Cisneros, Sandra. (2002) Caramelo or Puro Cuento. Londres: Bloomsbury (texto origen -TO-), y Cisneros, Sandra. (2003) Caramelo o puro cuento. Traducción de Liliana Valenzuela. Barcelona: Seix Barral (texto meta-TM-). mos en el siguiente apartado, «she tried to find parallels, to things, where if something was in Spanish she might put English in the text, so you would get the sense of the two languages" (Birnbaum, 2002). De hecho, la misma traductora da cuenta de sus decisiones en la Nota a la traducción: el revés del bordado, que acompaña la versión española de Caramelo or Puro Cuento. La dificultad de la traducción requirió un esfuerzo adicional de Valenzuela, y el conocimiento exacto de la realidad chicana que poseía fue esencial para lograr el resultado final.

Caramelo or Puro Cuento es una obra donde abundan los elementos culturales y donde el cambio de código, como parte de éstos, se encuentra presente por doquier. A continuación, se examinarán los métodos utilizados por la autora para entremezclar inglés y español en el mismo discurso, y, a su vez, las estrategias traductológicas a las que recurre Valenzuela para encontrar equivalencias a esta característica lingüística.

\section{ESTRATEGLAS DE CAMBIO DE CÓDIGO Y SU TRADUCCIÓN}

Según Sandra Cisneros, la incorporación del español en su obra le permite crear nuevas expresiones en inglés, decir cosas que no se han dicho antes, y añadir «a new spice [...] to the English language» (Dasenbrock, citado por Pearce, 1999). Este objetivo lo logra, por ejemplo, traduciendo literalmente expresiones o refranes al inglés: "God squeezes, but $\mathrm{He}$ doesn't choke» (TO: 119). Liliana Valenzuela afirma que «este recurso de traducir literalmente expresiones muy mexicanas al inglés produce un efecto de extrañeza en el lector que lee la obra en inglés, y le abre un universo de sensibilidades y de formas de pensar muy distintas a las suyas» (TM: 541). Ésta constituye una de las características de una novela plagada de términos e interjecciones en español, y traducciones y explicaciones en inglés. En opinión de 
la autora, "all the expresiones in Spanish when translated make English wonderful" (Dasenbrock, citado por Pearce, 1999) (el énfasis es mío). Ésta supone una actitud similar a la adoptada por los defensores del spanglish.

$\mathrm{Al}$ analizar las situaciones y contextos en los que se revela el cambio de código en Caramelo or Puro Cuento, se pueden establecer una serie de pautas que la autora sigue para que la narración sea comprensible tanto para los lectores bilingües, como para los monolingües. De cualquier forma, esto no supone para Cisneros una preocupación especial. De hecho, toma por sentado de antemano que habrá pasajes que un lector anglosajón no comprenda, pero advierte que no sacrificará su obra por ello:

The readers who are going to like my stories the best and catch all the subtexts and all the subtleties, that even my editor can't catch, are Chicanas. [...] But I'm also very conscious when I'm writing about opening doors for people who don't know the culture. I try my best. I won't do it for the sake of an Anglo reader. There was one place in my story [la autora se refiere a La casa en Mango Street] when, for example, the grandmother cried because she found out I was going to "dar a luz". My editor finally put in the margins: "I think you need to translate this." I said, "I wish I could, but I don't know how without the seams showing, so we're going to have to leave it. People will have to use a dictionary. They can still get it" [...]. I'm not going to make concessions to the non-Spanish speaker. I will try my best everywhere else if it flows into the piece [...]. I'm not going to do that for the person who's monolingual, but I will try to weave it in in such a way in the rest of the story so they don't lose it. (Dasenbrock, citado por Pearce, 1999)

A pesar de esto, como veremos, el contexto que proporciona la obra suele ser suficiente para que un lector anglosajón entienda las incursiones en español, principalmente gracias a las estrategias utilizadas por Cisneros ${ }^{16}$.

\subsection{Término en español y su traducción en inglés $^{17}$}

Se trata éste de uno de los métodos más frecuentemente empleados a lo largo de la obra. Desde la perspectiva del cambio de código, esta estrategia se corresponde con la llamada constituent insertion de Poplack y Sankoff (1988: 1.178-1.179). Según esta técnica, se introduce en el discurso una palabra en un idioma distinto, aunque de la misma clase terminológica que la que correspondería en ese lugar de la frase (esto es, un sustantivo por un sustantivo, un adjetivo por un adjetivo, etc.). También se adivina en este caso el procedimiento de message qualification de Gumperz (1982: 79), por el que se especifica, en un idioma, cierta información ya proporcionada en otro código. Por otra parte, si tomamos la estrategia desde la óptica de los métodos de traducción, se correspondería con la autotraducción interpersonal, es decir, traducir de una lengua a otra algo que ya se ha dicho, con el

\footnotetext{
I6 Algunas de las declaraciones que confirman esta afirmación, desde el punto de vista de la recepción de la obra, son: «Ms. Cisneros's writing is often described by her readers as poetic yet accessible, even when she sprinkles it with Spanish words that go untranslated. (They make sense to English speakers in context)» (Navarro, 2002); "Fluency in Spanish is helpful but not required" (Kline, 2002); "Most [chapters] are laced with Spanish (some of it is translated, and context makes most of the rest clear)» (Bancroft, 2002); «Spanish is sprinkled liberally throughout the book, but most of it is easily figured out by context" (Sandoval, 2002)

I7 Martín Rodríguez (2000: 257) considera que, en general, en la literatura chicana, «este tipo de traducción directa revela un deseo de llegar a un público no-hispanohablante (incluyendo en dicho grupo a numerosos lectores chicanos). Pero el efecto de repetición que produce la traducción directa de lo que dice el personaje se convierte en un lastre para el lector bilingüe que reduce sobremanera su apreciación del texto». Personalmente, no estoy de acuerdo con esta apreciación. Quizás debido a mi condición de traductora, este tipo de estrategias me resultan sumamente interesantes desde el punto de vista lingüístico, y no considero que mermen mi valoración positiva del texto. $\mathrm{Al}$ contrario, creo que suponen un aliciente más para apreciar la presencia de dos culturas y el bilingüismo que caracterizan a la novela.
} 
objetivo de que otros lo entiendan (Harris y Sherwood, citados por Toury, 2004: 308).

La traducción del término español suele aparecer en la obra de dos formas: en otro lugar del texto, aunque no lo suficientemente alejado como para quedar fuera del contexto, o lindando con la palabra o expresión en español.

El texto meta reproduce estas traducciones sólo en los casos en los que no resultan excesivamente repetitivas al trasladarlas al español. Si la repetición es sutil y se encuentra suficientemente alejada de la palabra en cuestión, Valenzuela suele optar por dejarla inalterada en la versión, como ocurre en el siguiente ejemplo:

«- $[\ldots]$ What's in there?

-Where?

- In there. Inside that.

- ¿El ropero? Oh, lots of things. Lots.

Would you like to see?

The grandfather walks over to the walnut-wood armoire [...]» (TO: 57).

$\ll-[\ldots]$ ¿Qué hay ahí?

- ¿Dónde?

- Ahí. Allá adentro.

- ¿El ropero? Ah, muchas cosas.

Muchas. ¿Te gustaría ver?

$\mathrm{El}$ abuelo camina hacia el ropero

de nogal [...]» (TM: 79).

\section{Del mismo modo:}

«-El cuarenta y uno, Father shouts to his mother, to our mother, to anyone who will listen. This means the Grandmother should punch the remote on her bedroom portable to channel 41 , and Mother should turn the dial to 41 on her kitchen TV [...]» (TO: 332).

«-¡El cuarenta y uno!- papá le grita a su mamá, a nuestra mamá, a quienquiera que lo escuche. Esto quiere decir que la abuela debe oprimir el control remoto de su tele portátil de la recámara al canal 41 , y mamá debe cambiar el disco selector al 41 en su tele de la cocina [...]» (TM: 404).
La autora también introduce las traducciones dentro de la redacción del texto. Este método es una muestra más de su habilidad para crear un contexto lingüístico destinado al lector monolingüe, sin que resulte repetitivo. Esto último lo logra en el siguiente ejemplo, uno de los más significativos de este tipo, cambiando el orden de los adjetivos en la traducción:

"It was the cultural opinion of the times that men ought to be feos, fuertes, $y$ formales. Narciso Reyes was strong and proper, but, no, he wasn't ugly" (TO: 103).

«Era la opinión cultural de esos tiempos que los hombres debían ser feos, fuertes y formales. Narciso Reyes era fuerte y formal, pero, no, no era feo» (TM: 132).

En cualquier caso, y por si quedaba alguna duda en el lector, los tres adjetivos se repiten, por orden, en otro lugar del texto, aunque esta vez se omiten en la traducción:

"[...] Feo, fuerte y formal. A man has got to be ugly, strong and proper, he kept repeating to himself in order to keep from buckling under" (TO: 129).

«[...] Un hombre tiene que ser feo, fuerte y formal, seguía repitiéndose a sí mismo para evitar que le fallaran las rodillas» (TM: 162).

Lo que, al fin y al cabo, se hace en estos ejemplos, es enmascarar la traducción del término dentro de una frase explicativa. En el siguiente ejemplo, observamos dos tipos de traducción. En el caso de rico, claramente indicada; en el caso de pobre, insertada en la oración posterior:

«[...] on a block where everyone knows Uncle Fat-Face by his Italian nickname, Rico, instead of FatFace or Federico, even though "rico" means "rich" in Spanish, and Uncle is always complaining he is pobre, 


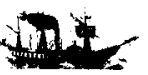

48 pobre. - It is no disgrace to be poor, Uncle says, citing the Mexican saying, -but it's very inconvenient" (TO: 11).

«[...] en una cuadra donde todos conocen a tío Chato por su apodo italiano, "Rico", en lugar de Chato o Federico, aunque "rico" quiere decir "rich" en inglés, $y$ tío siempre se queja de que es pobre, pobre. "No es desgracia ser pobre -dice tío, mencionando el dicho mexicano-, pero es muy inconveniente"» (TM: 24).

En otros pasajes, la traducción sigue al término en español y, por tanto, se elimina en el texto meta, ya que la repetición resulta innecesaria. Este tipo de recurso es también muy abundante en la obra:

"The diagnosis was a collapsed lung, but the real cause of Narciso Reyes' trouble, as Regina never tired of explaining, was susto, fear, a Mexican malaise responsible for centuries of harm» (TO: 132).

«El diagnóstico fue un colapso de pulmón, pero la verdadera causa de los problemas de Narciso Reyes, como Regina nunca se cansó de explicar, fue el susto, ese mal mexicano responsable de siglos de desgracias» (TM: $165)$.

\section{Otro ejemplo es:}

«I was a cupcake. ¿¿Quién te quiere? Who loves you?, he'd coon (TO: 232).

“Era un bombón. “¿Quién te quiere?”, susurraba" (TM: 283).

A veces, curiosamente, la propia autora reconoce la dificultad de trasladar un fragmento del español al inglés, sobre todo cuando se trata, como en este caso, de una alusión literaria:

«[... There's a poem by García Lorca we had to memorize once in school. It has a line that goes "Who will buy from me this sadness of white string to make handkerchiefs?"
Something like that. It sounds kind of goofy in English. $\dot{\zeta}$ Quién me compraria a mí, este cintillo que tengo y esta tristeza de bilo blanco, para bacer pañuelos?" (TO: 316 ).

"[...] Hay un poema de García Lorca que tuvimos que memorizar una vez en la escuela. Tiene un verso que dice: "¿Quién me compraría a mí, este cintillo que tengo y esta tristeza de hilo blanco, para hacer pañuelos?" Algo así. Suena medio bobo en inglés. Who will buy from me this sadness of white string to make bandkerchiefs? (TM: 385).

En la traducción se cambian de posición ambas frases, sin que esto suponga una modificación en el texto. En este caso, mientras Cisneros comienza traduciendo el verso antes de dar la entrada al original, Valenzuela cita la versión española, y posteriormente, su traducción al inglés. De esta forma, mantiene el bilingüismo y, al mismo tiempo, da prioridad al español, como lo hace Cisneros con el inglés.

$\mathrm{Al}$ ser el cambio de código característica de la obra que se analiza, Liliana Valenzuela trata de reproducir la misma peculiaridad, añadiendo expresiones en inglés a su versión. De hecho, aprovechando la estrategia de traducción usada por Cisneros, la traductora realiza algunos cambios en su versión:

«[...]-I have siete bijos, Father begins, bragging about his seven "sons"» (TO: 80).

«[...]-Tengo siete hijos-comienza papá, presumiendo de sus siete "sons"»(TM: 104).

Obsérvese en este caso, también, el uso de las comillas. Esto puede servir para indicar al lector dónde se encuentra la traducción. Además, también cabe la posibilidad de que, en el texto meta, se trate de una estrategia para dar a entender que el discurso del padre está teniendo lugar en inglés. De hecho, no existe motivo aparente por el que la versión española añada la palabra «sons» (palabra que habría 
usado el padre en lugar de "children»). Cierto es que Valenzuela agrega numerosos términos $\mathrm{e}$ interjecciones en este idioma a lo largo de la obra, pero este caso es bastante sintomático, puesto que no se trata simplemente de vocativos, interjecciones o frases completas, sino de un sustantivo, y a modo de traducción. A este respecto, Sandra Cisneros ya advierte que, en su propia obra, «now I'm learning how you can say something in English so that you know the person is saying it in Spanish» (Dasenbrock, citado por Pierce, 1999). Por tanto, no es extraño que Liliana Valenzuela haya aprovechado esta circunstancia para trasladar el inglés a su traducción. De hecho, señala:

Para recrear este mundo de referencias culturales dobles para el lector en español, elegí el español mexicano en los diálogos, insertando algunos términos y expresiones en inglés que aun el lector monolingüe sea capaz de interpretar en la lectura con la ayuda de otras pistas. [...] [L]os narradores de Sandra Cisneros emergen de los márgenes y presentan un reto al discurso dominante. La traducción no puede menos que buscar la relación inversa pero proporcional, para invertir así también los códigos lingüísticos del español (TM: 541-543).

De esta forma, a lo largo de la obra, la traductora añade frases, términos e interjecciones en inglés, con el fin de crear en el lector español un efecto parecido al que la autora quiso crear en el anglosajón.

Observemos algunos ejemplos de modificaciones en el texto meta, con respecto al texto origen. En el siguiente, nos encontramos claramente ante un cambio de código, motivado por el escaso conocimiento que la niña protagonista posee de ambas lenguas. $\mathrm{La}$ traducción contiene más oraciones en inglés que el texto origen en español. En concreto, el original sólo cita tres oraciones, mientras que la versión reproduce once. La traductora utiliza la circunstancia de la poca competencia lingüística de la niña para explotar la mezcla español-inglés que su texto podría haber perdido en otros pasajes ${ }^{18}$. Del mismo modo, desde el punto de vista de la adquisición de idiomas, resulta interesante el último párrafo, en el que Lala, la narradora, explica que su padre la llama cielo, cuando quiere decir my heaven (ésta última explicación se omite en la versión española, puesto que se encuentra en la inglesa por meras razones de contexto lingüístico). Sin embargo, cuenta, su abuelo traduce la palabra a una de las dos acepciones de cielo, precisamente, a la que no corresponde:

«I scramble downstairs to tell everyone, only I don't know the words for what I want to say. Not in English. Not in Spanish.

-The wall has fallen, I keep saying in English.

-What?

-Upstairs. In the big dining room. The wall fell. Come and see.

-What does this kid want? Go see your mother.

-It's that the wall has fallen.

-Later, sweetie, not now, I'm busy.

-The wall in the dining room, it came down like snow.

-How this child loves to be a pest!

-What is it, my queen? Tell me, my heaven.

-La pared de arriba, es que se cayó. Ven, Papá, ven.

-You go, Zoila. You're the mother.

$-i$ Ay! Always, always I'm the mother when you can't be bothered. All right, all right already. Quit pulling at me, Lala, you're going to rip my dress.

$[\ldots]$

-Everybody, quick! The ceiling's fallen!

- - Se cayó el cielo raso!, Father says.

And then it is I learn the words for what

I8 Lo que en realidad está aplicando Valenzuela con este tipo de cambios es una compensación con respecto a otras partes del texto en las que se ha perdido significado (o, en este caso, ciertas implicaciones lingüísticas de las que Cisneros quiso dotar a su obra).

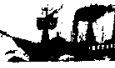


I want to say. "Ceiling" and "cielo". Cielo -the word Father uses when he calls me "my heaven". The same word the Little Grandfather reaches for when he wants to say the same thing. Only he says it in English. -My sky" (TO: 61).

«Bajo las escaleras como puedo para decirles a todos, sólo que no tengo palabras para lo que quiero decir. Ni en inglés ni en español.

- The wall has fallen-sigo diciendo en inglés ${ }^{19}$.

- What?

-Arriba. En el comedor grande. The wall fell. Come and see.

-¿Qué quiere esta niña? Go see your mother.

-Es que se cayó la pared.

-Later, sweetie, not now. I'm busy.

-The wall in the dining room, it came down like snow.

- ¿Qué latosa es esta niña!

- ¿Qué es, mi reina? Dime, mi cielo.

-La pared arriba, es que se cayó. Ven, papá, ven.

-Ve tú, Zoila. Tú eres la mamá.

-¡Ay! Siempre, siempre soy la mamá cuando no quieres que te frieguen. All right, all right already. Ya para de jalarme, Lala, vas a echar a perder mi vestido.

[...]

-Everybody, quick! The ceiling's fallen!

- ¡Se cayó el cielo raso! -grita papá.

$\mathrm{Y}$ entonces aprendo las palabras para lo que quiero decir. "Ceiling" y "cielo", la pala-

19 En este caso, tanto como en la versión inglesa, resulta innecesario especificar que se está hablando en inglés. Quizás en el texto origen la presencia de esta explicación esté hasta cierto punto justificada, puesto que no siempre que los personajes hablan en inglés significa que realmente estén hablando en ese idioma (véase anteriormente la cita de Sandra Cisneros al respecto). Martín Junquera (2000: 230) apunta al hecho de que este tipo de especificaciones están destinadas a llamar la atención del lector con respecto a la cuestión del idioma. Este tipo de explicaciones acerca del dialecto o lenguaje usado cuando una variación diatópica, diastrática diacrónica o técnica aparece en el texto, es lo que esta autora denomina the tag solution. bra que papá usa cuando me llama. La misma palabra que el abuelito trata de agarrar cuando quiere decir lo mismo. Sólo que él lo dice en inglés: “My sky" (TM: 82-83).

Siguiendo con el método de compensación, Valenzuela utiliza párrafos del texto origen carentes de incursiones en español, para introducir sus propias expresiones inglesas y su correspondiente traducción, tomando prestada la estrategia de Cisneros:

«-Shut up, stupid.

-I'm telling. Ma, Amor told me "shut up, stupid" " (TO: 12).

\section{«-Shut up, stupid.}

-Te voy a acusar. Ma, Amor me dijo "cállate, estúpida"» (TM: 26).

O, en este otro ejemplo:

«-Listen, you raise-heller, Mother continues $[\ldots]$ (TO: 85 ).

«-Mira, you raise-beller, vieja peleonera -mamá continúa [...]» (TM: 111).

\subsection{Español sin traducción}

Al igual que la estrategia anterior, la opción de dejar los términos en español sin incluir su traducción es una de las más numerosas de las utilizadas por Sandra Cisneros. En este caso, lógicamente, la versión traducida no suele añadir ni eliminar ningún elemento con respecto al texto original:

«-[...] Remember how she used to sing when she was just a baby? , Qué maravilla! She was just the same as Shirley Temple. [...] Still in diapers but there she was singing her heart out, remember? [...]" (TO: 58).

«-[...] Se acuerdan cómo cantaba cuando era sólo una criatura? Qué maravilla. Era igualita a Shirley Temple. [...] Todavía en pañales pero ahí andaba cantando con todas 
sus ganas, ¿se acuerdan? [...]» (TM: 80).

Como ya comentaba con anterioridad, cuando una palabra en español queda sin traducción ni explicación en el inglés, Sandra Cisneros suele aportar un contexto que clarifique su significado. En este caso, las dos frases posteriores a la exclamación en español no dejan lugar a dudas para un lector monolingüe de que se trata de una expresión positiva en cuanto a las habilidades de canto de la niña.

El mismo caso se da en el siguiente ejemplo, donde el contexto deja claro, si no el significado concreto del término español, sí la idea que pretende transmitir:

«Just as Narciso stepped in the door [...] [a] bowl whizzed past his head, shattering in a hail of milk and clay shards.

- Ay, escuincles! Aunty Fina said in a tone both disgusted and resigned. She dabbled at Narciso with a diaper.-I am sorry. [...] But you know how children are, right? [...]" (TO: 104).

«Al momento en que Narciso puso un pie en la puerta, [...] [u]n tazón pasó zumbando por su cabeza haciéndose añicos en una lluvia de leche y pedazos de barro.

- ¡Ay, escuincles! -dijo tía Fina en un tono a la vez indignado y resignado. Secó a Narciso con un pañal-. Lo siento. [...] Pero ya sabe cómo son los niños, ¿verdad? [...]» (TM: 133).

El último ejemplo de este tipo incluye, además de un contexto semejante al anterior, la similitud grafológica de la palabra principal, borror, en ambos idiomas:

"- $A y$, ay, qué borror! She keeps muttering when she finally returns to Father and me.Get me out of this inferno of Indians [...]” (TO: 79).

"-¡Ay, ay, qué horror!-sigue refunfuñando cuando finalmente regresa a donde estamos papá y yo-. ¡Sáquenme de este infierno de indios! [...]» (TM: 103).

\subsection{Otras estrategias}

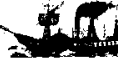

A pesar de que, desde el punto de vista traductológico, los anteriores son los métodos más interesantes usados por Cisneros a lo largo de la obra para introducir el español en el discurso inglés, existen otras incursiones de nuestro idioma que merece la pena reseñar aquí. En la mayoría de las conversaciones que mantienen los personajes de la obra, es frecuente hallar multitud de interjecciones, vocativos y onomatopeyas emitidas en español.

\section{Interjecciones $^{20}$}

Las expresiones precedidas por iAy', caray o válgame Dios, entre otras, son frecuentes en Caramelo or Puro Cuento, así como otras más propiamente mexicanas como ándale o a poco. Éstas no encuentran traducción ni en el texto origen, ni en el meta. Valenzuela, sin embargo, se cerciora de compensar esta pérdida en su traducción, añadiendo interjecciones propias del inglés. De esta forma, si una expresión queda sin traducción en la versión en español, como en el siguiente caso:

"-Listen to me, I'm talking to you. I said I'm talking to you!

$-A y$, caray, I'm trying to watch television [...]” (TO: 414),

"-Óyeme, te hablo. ¡Dije que te hablo!

-Ay, caray, estoy tratando de ver la televisión [...]» (TM: 505),

la traductora lo compensa en otro lugar del texto (no necesariamente cercano a aquél donde se ha producido la pérdida), dejando sin

20 Esta estrategia se corresponde con el tag-switching que menciona Romaine (1989: 122), que consiste en la introducción de coletillas en un idioma diferente al del discurso. Gumperz (1982: 77) también cita las interjecciones dentro del cambio de código. 
traducir alguna interjección inglesa:

«-Honey, that chair's not to sit on. Never sit on Aunty's bed, sweetie [...]. Put all the throw pillows back exactly like you found them when you leave, okay?» (TO: 14).

"Honey, esa silla no es para sentarse, nunca te sientes en la cama de tu tía, cariño, [...]. Pon todos los cojines de adorno exactamente como los encontraste cuando te vayas, okey?" (TM: 28).

\section{Vocativos}

La introducción de vocativos en la versión original de la obra contribuye, asimismo, a dotar a la novela de un entorno bilingüe y bicultural. Del mismo modo que ocurre en el caso de las interjecciones, en la traducción se pierden estas connotaciones, $y$, al igual que en el apartado anterior, Valenzuela subsana las pérdidas a lo largo del texto meta.

En este caso, sin embargo, y al contrario de lo que ocurría con las interjecciones, sí existen traducciones de los vocativos en la versión original.

Por ejemplo, vieja es el primero que se presenta en la obra. De la página 6 a la 8 aparece tres veces, pero hasta la última no encontramos su traducción: fun.

«-[...] Ya verás. You'll see, vieja, it'll be

-And stop calling me vieja, Mother shouts back.-I hate that word! I'm not old, you're mother's old" (TO: 8). tir.

«-[...] Ya verás, vieja, nos vamos a diver-

-Y no me llames vieja-mamá le contesta a gritos-. ¡Me choca esa palabra! No soy vieja, la que es vieja es tu mamá» (TM: 21).

Otros pueden ser fácilmente reconocidos por el contexto que proporciona la autora. En el momento en el que tiene lugar la siguiente frase, ya se ha mencionado que la madre de los tres hermanos que viajan a México es la $A w f u l$ Grandmother (la abuela enojona). Por lo tanto, el significado de mijos queda especificado en la frase donde esta palabra aparece por primera vez, a pesar de que, en este caso, no lo haga bajo la forma de vocativo:

"It's the Awful Grandmother's idea that her mijos drive down to Mexico together" (TO: 9).

«Fue idea de la abuela enojona que sus mijos manejaran juntos a Méxicon (TM: 22).

De cualquier forma, Cisneros especifica su traducción la siguiente ocasión en la que la palabra aparece en la novela:

"The Awful Grandmother calls Father mijo. Mijo. My son. - Mijo, mijo. [...] She uses the Spanish word bijos, which means sons and children all at onces (TO: 29).

Liliana Valenzuela, por su parte, aprovecha esta definición lingüística para introducir términos en inglés:

"La abuela enojona le dice "mijo" a papá. "Mijo, mijo." [...] Usa la palabra en español "hijos", que quiere decir sons y cbildren a la vez» (TM: 45).

Mi rey también se explica con su correspondiente traducción, que desaparece en el texto meta: 196).

"Mi rey, my king, she cooed [...]" (TO: «Mi rey-le canturreaba [...]» (TM: 241).

Otros vocativos presentes en la versión son señora, señor, pobre (y sus diminutivos: pobrecito, pobrecita), compadre, chulito, precioso, así como insultos: canalla, atrevido, viejo, metiche, aprovechado, etc.

En cuanto al texto de la traducción, uno de los vocativos que aparecen con más frecuencia es brotber, que en la mayoría de las ocasiones 
también se encuentra presente en la versión original:

«Oh, brother, Lolo groans.-I think I'm getting carsick» (TO: 242 ).

«Oh, brother -se queja Lolo-. Creo que me estoy mareando» (TM: 295).

Otros vocativos ingleses en el texto meta son my friend, baby, boney, etc.

También podemos encontrar confusiones en cuanto al sonido de las palabras que recuerdan a otros vocativos:

«I'm talking to you, Mother continues.- $T e$ bablo. Which sounds like the Spanish word for Devil.

-Ma, why you calling him diablo? I say to be funny» (TO: 235).

«-Te hablo -continúa mamá-, te hablo.Que suena como la palabra que significa devil en español.

-Ma, ¿por qué le dices diablo? -digo para hacerme la chistosa" (TM: 287).

Como se observa, Valenzuela ha mantenido la confusión lingüística añadiendo la palabra devil a la traducción.

\section{Onomatopeyas}

El caso de las onomatopeyas es especialmente complicado desde la óptica traductológica. Como el resto de elementos mencionados, forman parte de los factores culturales presentes en esta obra. La cultura a la que aluden, sin embargo, es mayoritariamente la norteamericana. Su traducción no parece haber planteado excesivos problemas a Valenzuela, que sabiamente opta por adaptarlas y sustituirlas por sus equivalentes españolas ${ }^{2 \mathrm{~T}}$ :

«[...] Then the gates shut with a terrible clang like in prison movies» (TO: 62).

21 Caso distinto es el de la traducción audiovisual, donde, además de por razones de doblaje o de limitaciones con respecto a la imagen que se ofrece, las onomatopeyas ingle-
«[...] Luego las rejas se cierran con un gran cuas como en las películas de cárceles». (TM: 85).

Esto también se puede comprobar en el siguiente ejemplo:

«[...] The México City Monte Carlo Grand Prix. Vroom-vroom. Cars howl, VW taxis [...] putt putt, a police siren yowls, [...] horns toot toot an impatient trumpet tap $[\ldots] »(\mathrm{TO}: 384)$.

"[...] El Grand Prix de Monte Carlo de la Ciudad de México. Rrrum, rrrum. Los coches aúllan, los taxis Volkswagen [...] hacen chuc, $c b u c$, la sirena de una patrulla maúlla, [...] las bocinas hacen pip pip como un impaciente toque de trompeta [...]» (TM: 466).

En otras ocasiones, la onomatopeya inglesa se convierte en un verbo español:

«Pulling shut the green iron gates with a clang $[\ldots] »$ (TO: 70).

"Cerrando las rejas de fierro verde con gran estruendo [...]» (TM: 94).

A veces, se mantienen igual, o su grafología se adapta a la española:

«[...] clip-clop of horses, hum of electric trams, hoarse, sad cries of the mules hauling streetcars, [...] click-click-click of hard boots [...]" (TO: 109).

sas están sustituyendo a las españolas a pasos agigantados. Xosé Castro (2003) considera que "a los buenos traductores audiovisuales se los distingue, entre otras muchas cosas, por su forma de traducir las onomatopeyas, interjecciones y otras muchas expresiones casi gestuales que tienen una enorme carga expresiva». Además, confirma que "cada vez oímos más onomatopeyas e interjecciones sin traducir en cine y televisión: iak, ups, yija (en inglés, yuk, oops, yeeha) en lugar de sus traducciones españolas (puaj/aj; janda!/uy/caray; ¡bien!/iguay!» (Castro, 2003). Si no se pone remedio a esta situación, sin embargo, puede que en un futuro encontremos estas expresiones híbridas en algún texto literario traducido. 
"[...] el clip clop de los caballos, el zumbido de los tranvías eléctricos, los gemidos roncos $\mathrm{y}$ tristes de las mulas jalando las carretas, [...] el clic, clic, clic, de las botas duras [...]" (TM: 140).

A pesar de que las onomatopeyas sean, en su mayoría, en inglés, también es posible hallar expresiones calcadas del español, que no son sino un ejemplo más de cambio de código en la redacción:

"[...] Papá. Father's name in Spanish with the accent on the end, papa. The End. Tan tan. Like the notes at the end of a Mexican song that tell you to applaud» (TO: 393).

«[...] Papá. El nombre de papá en español con el acento al final. Papá. El fin. Tan tan. Como las notas al final de una canción mexicana que te indican que aplaudas» (TM: 478).

Resulta curiosa la falta de acento en el segundo tan en la versión traducida, que puede deberse a un error tipográfico o bien a una adaptación del elemento al inglés, para conseguir el mismo efecto que se pretendía en el texto origen.

\section{Errores fonéticos}

Uno de los rasgos que caracterizan a cada nuevo hablante de una segunda lengua es su acento, su modo de pronunciar los sonidos que difieren de aquellos que está acostumbrado a usar en su lengua materna. En su afán de recrear un mundo protagonizado por chicanos, Sandra Cisneros no ha dejado de incluir estos errores de pronunciación a lo largo de su obra. Para ello, ha transcrito las palabras inglesas tal y como sonarían si un hablante español sin demasiado conocimiento fonológico de esa lengua las dijera.

Precisamente, existe incluso un capítulo que se titula «Spic Spanish?» y que Liliana Valenzuela traduce como «Espic espanish?», añadiendo la $e$ inicial que caracteriza a la pronunciación que el hispanohablante realiza de la $s$ liquida inglesa. Este capítulo explica el aprendizaje de inglés del padre de la protagonista, $y$ es uno de los más prolíficos en cuanto a ejemplos de este tipo:

«Father sent away for the Inglés Sin Stress home course in English. He practiced, when speaking to his boss, -Gud morning, ser. Or meeting a woman, $-J a ́ u d u$ iu du? If asked how he was coming along with his English lessons, -Very uel, zanc iüm (TO: 208).

"Papá mandó pedir el curso Inglés Sin Stress para estudiar en casa. Practicaba cuando hablaba con su jefe: "Gud morning, ser." $\mathrm{O}$ al conocer a una mujer: "Jaú du iú du?" Si le preguntaban cómo le iba con sus clases de inglés: "Beri güel, zenc iừ (TM: 255).

Obsérvese el cambio en la versión traducida, adaptando las "transcripciones» a la verdadera forma de pronunciar del hispanohablante. Del mismo modo, véase cómo la traductora aprovecha este capítulo para incluir errores fonéticos que no se recogen en el original, pero que sirven a la traductora para compensar otras pérdidas del texto:

"But his English was odd to American ears. He worked at his pronunciation and tried his best to enunciate correctly. $-\mathrm{Sir}$, kindly direct me to the water closet. -Please what do you say? -May I trouble you to ask for what time is? - Do me the kindness to tell me how is. When all else failed and Father couldn't make himself understood, he could resort to, -Spic Spanish?" (TO: 208).

«Pero su inglés sonaba raro a los oídos americanos. Se esforzaba en su pronunciación y hacía lo posible por enunciar correctamente. "Ser, kaindly direct me to de water closet." "Pliis, guat du iu say?" "May I trouble iù to ask for guat time it is?" "Du me de kaindness to tel me jau is." Como último recurso y cuando papá no podía hacerse entender, recurría a 
“Espic Espanish?"»(TM: 255).

Otra compensación en la traducción es la siguiente. Como vemos, Valenzuela mantiene el mismo tipo de transcripción que Cisneros:

"-Sir, I am of the same opinion, said Inocencio. He could not think what else to say. And even if he had, he couldn't say it.

-Let's drink to you, Inocencio, said Mr. Dick.-And to your happy race.

-You are very kind, sir.[...]» (TO:211).

«-Ser, I am of de seim opinion-dijo Inocencio. No se le ocurría qué más decir. Y aun si se le hubiera ocurrido, no habría podido decirlo.

-Brindemos por ti, Inocencio -dijo míster Dick-, y por tu raza alegre.

-Iú are bery kaind, ser. [...]» (TM: 259).

\section{Incursiones culturales, lingüísticas $y$ tipograficas}

Caramelo or Puro Cuento está compuesto por un total de 86 capítulos, divididos en tres partes. Muchos de esos títulos están escritos en español, otros en inglés, $y$, de entre estos últimos, muchos son traducciones literales de refranes o expresiones mexicanas o del español: The Little Mornings («Las mañanitas o the little mornings", en la traducción), Neitber with you, nor without you ("Ni contigo ni sin ti"), God gives almonds («Dios le da almendras», comienzo de un refrán que se cita al final del capitulo: God gives almonds to those without teeth, «Dios le da almendras a quien no tiene dientes") y $M y$ cross («Mi cruz»). Además, existen dos capítulos con la traducción del título incluida dentro de éste: A poco-You're kidding ("A poco») y Mexican on both sides or metiche, mirona, mitotera, hociconaen otras palabras, cuentista-Busybody, ogler, liar, gossip, troublemaker, big-mouth -in other words, storyteller (el título español no recoge la traducción al inglés). Sólo el nombre de uno de los capitulos permanece en inglés en la versión de
Liliana Valenzuela (además de Espic espanish?, que ya he mencionado): No worth the money, but they belp a lot. Se mantiene en este idioma, porque la expresión en sí es una traducción de «El dinero no vale, pero ayuda mucho».

Por otra parte, como es frecuente en este tipo de textos, los elementos relativos a la gastronomía ocupan también una parte importante de la obra:

"It was the room above the store that sold popcorn, candy, gelatines, and fresh fruit drinks - horchata, chia, tamarindo, piña, jamaican (TO: 169).

«Se trataba de la recámara encima de la tienda que vendía palomitas de maíz, dulces, gelatinas y aguas frescas de frutas: horchata, chía, tamarindo, piña, jamaican (TM: 211).

Otro ejemplo es:

«[...] a basket of pan dulce; Mexican sweet bread; hotcakes with honey; [...] frijoles with fresh cilantro; molletes; or scrambled eggs with chorizo; eggs a la mexicana with tomato, onion, and chile; or buevos rancheros. [...] $[$ F]resh-baked rusty bolillos [...]; carne asada; [...] tortillas» (TO: 17).

[...] una canasta de pan dulce; bot cakes con miel de abeja; [...] frijoles con cilantro fresco; molletes; o huevos revueltos con chorizo; huevos a la mexicana con jitomate, cebolla $y$ chile; o huevos rancheros. [...] [B]olillos crujientes recién horneados $[. .$.$] carne asada; [. .$. tortillas" (TM: 34 ).

Mientras que en el primer caso, la traductora no realiza ningún cambio, no ocurre lo mismo con este último, en el que se mantiene en inglés uno de los elementos: hotcakes. Resultaba complicado añadir más términos ingleses a un párrafo tan marcadamente cultural.

La incursión del español en la obra se puede apreciar, asimismo, en las traducciones literales de otros elementos españoles. De esta forma, es 
frecuente encontrarse con it's that («es que»), an anybody (con el sentido de «una cualquiera») o expresiones como qué microwave ni qué nada (TO: 121). Los primeros casos suelen traducirse con su correspondiente versión española. El último, sin embargo, se reproduce idénticamente en la traducción, enfatizando, en ese caso, el término inglés, al contrario que en la versión original (TM: 153).

Por último, y aunque la tipografía no puede ser considerada cambio de código, sí se debe dejar constancia del uso que hace Cisneros de los guiones que introducen los diálogos en su obra (tipografía propia del español), en lugar de las tradicionales comillas inglesas. De cualquier forma, como se puede comprobar en la mayoría de los ejemplos precedentes, no utiliza un sistema exclusivamente español, sino que, una vez más, la autora nos sorprende con lo que podría tratarse de un "cambio de código tipográfico", donde se mezclan elementos de ambos idiomas.

\section{CONCLUSIONES}

Si la tarea de traducir un texto con marcado contenido cultural supone un reto para cualquier traductor, no lo es menos el que plantea aquél que, además, contiene una mezcla lingüística que lo enriquece, pero que también dificulta su traducción.

Al comenzar la realización de este trabajo, la naturaleza del cambio de código me interesaba personalmente, en especial por su implicación lingüística. Aunque en un principio se tiende a identificar este fenómeno con el spanglish, desde el momento en el que se profundiza en su estudio, se intuye que no se trata de spanglish, entendido en sentido estricto. En este caso, supone un rasgo de identificación social y cultural extremadamente importante para los chicanos. Estudiar el cambio lingüístico de esta comunidad, supone, para ellos, estudiar la "lengua chicana», y, por ende, adentrarse en el complicado mundo de las implicaciones y equivalencias culturales.

Como ya señalaba al comienzo, este fenómeno no es exclusivo de la comunidad chicana. De hecho, es probable que en un futuro no muy lejano asistamos a la aparición de mezclas similares entre idiomas, propiciada por la inmigración y el contacto directo entre hablantes de distintas lenguas que cohabitan en una misma sociedad.

El libro que he tomado como pilar de mi trabajo constituye un ejemplo perfecto en este sentido. En él, Sandra Cisneros refleja una cultura y una forma de vida que envuelve a todo el que lee la obra.

Para recrear el cambio de código que utilizan los personajes de la novela, la autora ha empleado, principalmente, cinco estrategias. Sólo una de ellas, sin embargo, parece especialmente pensada para Caramelo or Puro Cuento, ya que la aparición de la traducción de un término en español no es, precisamente, un elemento que suela acompañar a los cambios de códigos en pleno discurso. Este método está obviamente enfocado al público anglosajón. Además, en los casos en los que la naturaleza innata del cambio de código (la incursión del español, sin traducción), aparece en el texto, la autora recurre a la ayuda del contexto para clarificar su significado. También se ha comprobado que existe traducción en el caso de los vocativos, y contexto, en el de las onomatopeyas. Algo distinto ocurre con las interjecciones, que reflejan el cambio de código en su estado más puro: es decir, en su mayoría, aparecen sin traducción y sin contexto que las acompañe. Además, Cisneros utiliza la traducción literal de expresiones y refranes, y una tipografía que recuerda a la del español, para enfatizar, desde el inglés, la omnipresencia de nuestro idioma en el texto, aun de forma encubierta.

La traducción, en consonancia con la estrategia seguida por Cisneros, logra recrear este mundo de bilingüismo añadiendo, a lo largo de 
la obra, términos, expresiones y frases en inglés, a modo de compensación. Las estrategias que utiliza Liliana Valenzuela son las mismas que las usadas por la autora. Sin embargo, su empleo varía en función de la redacción del texto meta. De esta forma, no siempre que se incluye cambio de código en el original, lo encontramos en la traducción, y viceversa. Decididamente, el hecho de que autora y traductora trabajaran juntas en las etapas previas a la realización de la versión en español, ha sido primordial para el resultado final.

Aquél que se vale del cambio de código como sistema de comunicación, es una persona que vive entre dos lenguas y dos culturas $y$, por tanto, hace uso de un elemento que contribuye a la convivencia entre ellas. Así, traspasar este fenómeno al mundo literario, supone, por naturaleza, encerrarlo dentro de una serie de reglas, con el fin de hacer llegar su esencia a aquellos que no lo comparten. Es tarea, pues, del autor y del traductor de la obra, establecer una serie de estrategias que hagan posible este propósito. La factibilidad de éstas se verá reflejada en la recepción del texto, tanto entre el público origen como entre el meta.

$Y$ para finalizar, se debe resaltar que Sandra Cisneros, experta conocedora del cambio de código y de las dos lenguas y culturas en contacto, ha conseguido integrar dentro del complicado mundo del bilingüismo tanto al lector anglosajón como al hispano. Liliana Valenzuela, por su parte, ha sabido plasmar la intención de la autora, utilizando los métodos del texto inglés y adaptándolos, hábilmente, a su versión española.

\section{RECIBIDO EN ENERO DE 2005}

\section{BIBLIOGRAFĹA}

Ammon, Ulrich, Norbert Dittmar y Klaus J. Mattheier (eds.). (1988) Sociolinguistics: An International Handbook of Language and Society. Berlin: Walter de Gruyter.

Baker, George C. (1975) "Social functions of language in a Mexican-American community". En Hernández Chavez, Eduardo, Andrew D. Cohen y Anthony F. Beltramo (eds.), pp. 170-182.

Bancroft, Colette. (2002) «A warmly woven tale». http://pqasb.pqarchiver.com/ spti$\mathrm{mes} / 199104521$.html?MAC $=\mathrm{a} 49612 \mathrm{bbc} 75952 \mathrm{e}$ 9825098f21baa8705\&did=199104521\&FMT = FT\&FMTS $=F T \&$ date $=$ \&author $=$ \&printforma $\mathrm{t}=\&$ desc $=\mathrm{A}+$ warmly + woven + tale + Series $\% 3 \mathrm{~A}+\mathrm{B}$ OOKS [13/10/2004].

Birnbaum, Robert. (2002) «Identity Theory: The narrative thread. Sandra Cisneros». http://www. identitytheory.com/people/birnbaum $76 . \mathrm{html}$ [10/10/2004].

Castro Roig, Xosé. (2001) “El ciberespanglish, el español comercial y el español neutro en la Red». http://www.elcastellano.org/span2.html [27/9/2004].

_- (2003) «La traducción de películas y audiovisuales». http://www.elcastellano.org/xcastro. html [12/11/2004].

Cisneros, Sandra. (1997) «My Purple House: Color is a Language and a Historyn. http://webenhanced.lbcc.edu/eng105sqr/handouts/mypurplehouse.html [18/11/2004].

. (2002) Caramelo or Puro Cuento. Londres: Bloomsbury.

- (2003) Caramelo o puro cuento. Traducción de Liliana Valenzuela. Barcelona: Seix Barral.

Díez Vegas, Francisco Javier. (1994) «Spanglish». http://www.ia.uned.es/ fjdiez/spanglish [27/9/2004].

Eguíluz Ortiz de Latierro, Federico. (2000) «Algunas reflexiones para entender la literatura chicana». En Morillas Sánchez, Rosa y Manuel Villar Raso (eds.), pp. 99-107.

Espinosa, Aurelio M. (1975) «Speech Mixture in New México: The Influence of the English Language on New Mexican Spanish». En Hernández Chavez, Eduardo, Andrew D. Cohen y Anthony F. Beltramo (eds.), pp. 99-114.

Flores, Lauro. (2000) "Identidad y cultura en la 
autobiografía chicana». En Morillas Sánchez, Rosa y Manuel Villar Raso (eds.), pp. 145-156.

González-Echeverría, Roberto. (1997) «Hablar spanglish es devaluar el español». http://www. elcastellano.org/clarin.html [27/9/2004].

Grosjean, François. (1995) "A psycholinguistic approach to code-switching: the recognition of guest words by bilinguals». En Milroy, Lesley y Pieter Muysken (eds.), pp. 259-275.

Guinn, Jeff. (2002) «Self possession». http:/www. twincities.com $/ \mathrm{mld} /$ pioneerpress/entertainment/books/4109420.htm?1c [30/10/2004].

Gumperz, John J. (1982) Discourse Strategies. Cambridge: Cambridge University Press.

Henríquez Betancor, María. (2000) «Chicanas' contemporary constructions of autobiographical texts». En Morillas Sánchez, Rosa y Manuel Villar Raso (eds.), pp. 173-181.

Hernández Chavez, Eduardo, Andrew D. Cohen y Anthony F. Beltramo (eds.) (1975) El lenguaje de los chicanos: Regional and Social Characteristics of Language used by Mexican-American. Arlington: Center for Applied Linguistics.

Ibarraran Bigalondo, Amaia. (2000) Novelistas cbicanas: hacia la creación de la identidad de la xicana. Universidad del PaísVasco.

Kline, Betsy. (2002) "Caramelo by Sandra Cisneros. Cisneros stirs up a Spanish treat". http://www.post-gazette.com/books/reviews/ 20021027cisneros1027fnp5.asp [27/10/2004].

Köppe, Regina y Jürgen M. Meisel. (1995) «Codeswitching in bilingual first language acquisition». En Milroy, Lesley y Pieter Muysken (eds.), pp. 276-301.

Latina: Magazine for Hispanic Women. http://www. latina.org [31/10/2004].

León Jiménez, Raquel. (2003) Identidad multilingüe. El cambio de código como símbolo de la identidad en la literatura chicana. La Rioja: Servicio de Publicaciones de la Universidad de La Rioja.

Lucio, Cristina. (2003) «El último crack americano. Sandra Cisneros: 'Antes sólo podía publicar en editoriales feministas'». http://www.el-mundo. es/elmundolibro/2003/05/23/ protagonistas/1053714008.html [30/10/2004].

Martín Junquera, Imelda. (2000) «The poetics of self-translation: the case of Rudolfo Anaya's Bless Me Ultima". En Morillas Sánchez, Rosa y
Manuel Villar Raso (eds.), pp. 227-234.

Martín Rodríguez, Manuel M. (2000) «Quién es el público y dónde se le encuentra? Literatura chicana y recepción». En Morillas Sánchez, Rosa y Manuel Villar Raso (eds.), pp. 253-261.

McQuien, Paul y Kim G. Hochmeister. «Sandra Cisneros". http://www.accd.edu/sac/english/ mcquien/htmlfils/cisneros.htm [18/11/2004].

Milroy, Lesley y Pieter Muysken (eds.). (1995) One speaker, two languages. Cross-disciplinary perspectives on code-switching. Cambridge: Cambridge University Press.

Morillas Sánchez, Rosa y Manuel Villar Raso (eds.). (2000) Literatura chicana: Reflexiones y ensayos criticos. Granada: Comares.

Muysken, Pieter. (1995) «Code-switching and grammatical theory», en Milroy, Lesley y Pieter Muysken (eds.), pp. 177-198.

Navarro, Mireia. (2002) "Telling a tale of immigrants whose stories go untold». http://www. nytimes.com/2002/11/12/books/12CISN.html [27/10/2004].

Newman, María. (2002) «Sandra Cisneros. Her new book, her new look». http://www.hispaniconline. $\mathrm{com} / \mathrm{magazine} / 2002 / \mathrm{sep} /$ CoverStory/index. html [25/11/2004].

Paternostro, Silvana. (2003) *The meaning of Spanglish. What happens when two languages become one?». http://msnbc.com/id/3069153 [27/9/2004].

Pearce, Richard. Multicultural Web-U.S. Fiction since 1950. (1999) «Reed Dasenbrock: Interview with Sandra Cisneros». http://acunix.whartonma. edu/ rpearce/MultiC_Web/Authors/Sandra_ Cisneros/sandra_cisneros.htm1 [22/10/2004].

Peñalosa, Fernando. (1975) «Chicano multilingualism and multiglossia». En Hernández Chavez, Eduardo, Andrew D. Cohen y Anthony F. Beltramo (eds.), pp. 164-169.

Poplack, Shana y David Sankoff. (1988) «Codeswitching", en Ammon, Ulrich, Norbert Dittmar y Klaus J. Mattheier (eds.), pp. 1.174-1.180.

Real Academia Española. http://www.rae.es [18/11/2004].

Romaine, Suzanne. (1989) Bilingualism. Oxford: Basil Blackwell.

Sandoval, Emiliana. (2002) "Cisneros serves up a big meal. Hispanic family tale has the past as 
salsa". http://www.freep.com/features/books/ cara22_20020922.htm [22/11/2004].

Seco, Manuel, Olimpia Andrés y Gabino Ramos. (1999). Diccionario del español actual. Madrid: Aguilar.

Toury, Gideon. (2004) Los estudios descriptivos de tra- ducción y más allá. Metodologia de la investigación en Estudios de Traducción. Traducción de Rosa Rabadán y Raquel Merino. Madrid: Cátedra. Valenzuela, Javier. «El vigor del 'spanglish'». http:// www.elcastellano.org/elpais.html [27/9/2004]. 